\title{
Expression, purification and characterization of human interferon- $\gamma$ in Pichia pastoris
}

\author{
DAN WANG, HUI REN, JING-WEI XU, PENG-DA SUN and XUE-DONG FANG
}

\author{
Department of General Surgery, The Second Hospital of Jilin University, Changchun, Jilin 130041, P.R. China
}

Received May 22, 2013; Accepted October 14, 2013

DOI: $10.3892 / \mathrm{mmr} .2013 .1812$

\begin{abstract}
Human interferon- $\gamma(\mathrm{hIFN}-\gamma)$ is a multifunctional protein known to possess immunoregulatory, antiviral and anticancer functions. In the present study, in order to explore the biological roles of hIFN- $\gamma$ and its mechanisms of action, IFN- $\gamma$ was cloned and expressed in Pichia pastoris (P. pastoris) under the control of alcohol oxidase promoter 1 (AOX1). The protein was secreted by two different signal peptides, the native secretion signal peptide of hIFN- $\gamma$ and the Saccharomyces cerevisiae $\alpha$ signal peptide. Following $96 \mathrm{~h}$ of methanol induction, Tricine-SDS-PAGE Coomassie staining, western blot analysis and $\mathrm{N}$-terminal protein sequencing revealed that the level of recombinant hIFN- $\gamma(\operatorname{rhIFN}-\gamma)$ secreted by the native secretion signal was barely detectable, while the $\alpha$ signal peptide secreted $\sim 300 \mathrm{mg} / \mathrm{l}$. rhIFN- $\gamma$ was purified by Vivaflow 200, SP Sepharose Fast Flow and Vivaspin $2 \mathrm{ml}$, yielding $>96 \%$ of a highly purified rhIFN $-\gamma$ preparation, with a specific activity of $1 \times 10^{7}-1.4 \times 10^{7} \mathrm{IU} / \mathrm{mg}$ protein as determined by an antiviral assay. The results demonstrated that the experimental procedures developed are capable of producing a large quantity of active rhIFN- $\gamma$ from $P$. pastoris.
\end{abstract}

\section{Introduction}

An interferon-like virus inhibitor, later designated as IFN- $\gamma$, was identified in phytohemagglutinin (PHA)-stimulated human leukocyte cultures (1). IFN- $\gamma$ is secreted by NK cells, $\gamma \delta \mathrm{T}$ cells and $\mathrm{CD} 8^{+} / \mathrm{CD} 4^{+} \mathrm{T}$ cells, which undergo activation by antigens, mitogens or alloantigens. IFN- $\gamma$ is important in the control of intracellular infection, immunoregulation, tumor development, hypersensitivity reactions, and autoimmune diseases $(2,3)$. Due to its numerous biological and pathological functions, human IFN- $\gamma(\mathrm{hIFN}-\gamma)$ is used extensively to treat

Correspondence to: Professor Xue-Dong Fang, Department of General Surgery, The Second Hospital of Jilin University, 218 Ziqiang Street, Changchun, Jilin 130041, P.R. China

E-mail: xuedongfang@yeah.net

Key words: protein expression, signal peptide, enzymatic cleavage, human interferon- $\gamma$, Pichia pastoris, secretion drug-resistant tuberculosis (4), atopic dermatitis (5) and hepatitis B infections (6).

Obtaining large quantities of pure hIFN- $\gamma$ is important for clinical studies and mechanistic investigations. Previous studies have reported that recombinant hIFN- $\gamma($ rhIFN- $\gamma$ ) was produced by an adenovirus (7), Escherichia coli (E.coli) (8-10), Saccharomyces cerevisiae (11), bicistronic baculovirus insects (12) and Chinese hamster ovary (CHO) cells $(13,14)$. However, complex manipulations, high culture expenses, low yields or low biological activity limit the use of these expression systems. The methylotrophic yeast, Pichia pastoris (P. pastoris), is frequently used as an expression system for the production of proteins. $P$. pastoris possesses prokaryotic features including clear genetic background, easy manipulation, high cell densities and low cost, and eukaryotic features including protein folding, post-translational modification and secretion.

In general, the secretion of a protein of interest into the culture medium is preceded by an $\mathrm{N}$-terminal extension of 3-60 amino acids, forming a signal peptide, which targets the propeptide of the endoplasmic reticulum followed by cleavage using membrane bound signal peptidases. hIFN- $\gamma$ has a native signal sequence of 23 amino acids, which directs its secretion from human lymphocyte cells. The Saccharomyces cerevisiae $\alpha$-mating factor $(\mathrm{MF}-\alpha)$ prepro sequence is widely used in $P$. pastoris cells to secrete heterologous proteins. MF- $\alpha$ consists of a signal sequence of 19 amino acids followed by a 66-residue sequence. The heterologous protein, fused with the signal sequence, is cleaved by the KEX 2 or STE13 gene product, at the site following the dibasic paired residues Lys-Arg or Glu-Ala, respectively. In this study, we compared the secretion level of rhIFN- $\gamma$ under the native secretion signal of hIFN- $\gamma$ versus the MF- $\alpha$ signal peptide, to achieve a native $\mathrm{N}$-terminal, and a higher quantity of a biologically active rhIFN- $\gamma$.

\section{Materials and methods}

Materials. $P$. pastoris X-33, pPICZaC vector, and Zeocin ${ }^{\mathrm{TM}}$ antibiotic were purchased from Invitrogen (Carlsbad, CA, USA). E. coli XL-Blue cells were purchased from Beijing Dingguo Biotechnology Company (Beijing, China). Restriction enzymes, DNA markers and protein markers were purchased from Takara (Dalian, China). The plasmid extraction kit was purchased from Feyou Biotechnology Co., Ltd. (Guangzhou, 
China). Primers and the coding sequence of hIFN- $\gamma$ were synthesized by Sangon Biotechnology Co. (Shanghai, China). The rabbit anti-IFN- $\gamma$ polyclonal antibody was purchased from Abcam (Cambridge, UK). Goat anti-rabbit IgG labeled with HRP was purchased from Beijing Dingguo Biotechnology Company. Vivaflow 200 polyethersulfone (PES) 50,000 molecular weight cut-offs (MWCO) and PES 10,000 MWCO and Vivaspin $2 \mathrm{ml}$ were purchased from Sartorius Stedim Biotech GmbH (Goettingen, Germany). SP Sepharose XL and Superdex G75 were purchased from GE Healthcare Bio-Sciences Co. (Piscataway, NJ, USA). Standard hIFN- $\gamma$ was purchased from Novoprotein (Shanghai, China). A Bradford protein assay kit was purchased from Beyotime (Shanghai, China).

Construction of expression vectors. The coding sequence of hIFN- $\gamma$, flanked with BspT1041 and KpnI, was synthesized by Sangon Biotechnology Co. based on the codon preference in $P$. pastoris, and the fragment between the $B s p \mathrm{~T} 1041$ and $K p n \mathrm{I}$ sites was inserted into pPICZ $\alpha \mathrm{C}$ at the same sites to generate pNhIFN. The optimized sequence encoding $h I F N-\gamma$ flanked with $B s p$ T1041 and KpnI was as follows: TTCGAAACGATGAAGTATACTAGTTAC ATCTTAGCCTTTCAATTGTGCATTGTTCTTGGTTC TTTGGGATGTTATTGTCAAGATCCATACGTTAAGGA GGCCGAGAACCTAAAGAAATACTTTAACGCCGGCCA TGACGTGGCAGATAATGGGACTTTCTCGTTTTTGGG TATTCTGAAAAATTGGAAGGAAGAGTCCGATAGAAA GATTATGCAATCCCAGATCGTAAGTTTCTACTTCAAG CTGTTTAAAAATTTCAAGGACGATCAGTCTATACAA AAATCAGTGGAGACCATTAAAGAAGACATGAACGTC AAGTTCTTCAACTCTAACAAAAAGAAGAGAGATGAT TTTGAAAAACTAACCAATTATTCTGTTACAGACCTGA ATGTTCAGAGAAAAGCTATACATGAATTAATCCAGG TCATGGCTGAACTTTCACCTGCTGCTAAAACGGGTA AACGAAAGAGATCACAAATGTTGTTTCGTGGAAGGA GAGCATCCCAATAAGGTACC. The recognition sequences for restriction enzymes BspT1041 and KpnI are underlined, and the bold shows the native secretion signal peptide of $h I F N-\gamma$.

To construct a plasmid containing the $h I F N-\gamma$ gene with an MF- $\alpha$ signal sequence containing a Kex 2 cleavage site (Lys-Arg), primers containing XhoI and XbaI recognition sites were used. In order to create a Kex 2 cleavage site upstream of the coding sequence of the mature hIFN- $\gamma$, bases coding for Lys-Arg were incorporated in the forward primer. Forward primer, 5'-GCCTCGAGAAGAGACAAGATCCATACG-3'; the recognition site for XhoI is underlined, and the bold letters signify the encoded region of the MF leader peptide of the Kex 2 cleavage site (Lys-Arg). Reverse primer, 5'-CCGTCTAGATTATTGGGATGCTCTCC-3'; the recognition site for $\mathrm{XbaI}$ is underlined, and the stop codon is in bold.

The polymerase chain reaction (PCR) was performed using the vector pNhIFN as a template, using 33 cycles of $94^{\circ} \mathrm{C}$ for $30 \mathrm{sec}, 55^{\circ} \mathrm{C}$ for $40 \mathrm{sec}$ and a final extension of $5 \mathrm{~min}$ at $72^{\circ} \mathrm{C}$. The product was digested with $\mathrm{XhoI}$ and $\mathrm{XbaI}$ and ligated into pPICZ $\alpha \mathrm{C}$ at the same sites, generating paIFN. pNhIFN and paIFN were verified by DNA sequencing.

Vector transformation into $P$. pastoris and screening with genomic PCR. pNhIFN and paIFN were isolated using an
Omega Bio-Tek plasmid mini kit (Norcross, GA, USA), linearized with SacI and transformed into the X-33 strain of $P$. pastoris according to the transformation protocol (Invitrogen). The transformed cells were selected on YPD (yeast extract, peptone and dextrose) containing Zeocin $(100 \mu \mathrm{g} / \mathrm{ml})$. Zeocin-resistant clones were isolated and the integration of $h I F N-\gamma$ was confirmed by PCR using AOX1 universal primers: forward, 5'-GACTGGTTCCAATTGACAAGC-3' and reverse, 5'-GCAAATGGCATTCTGACATCC-3'. Thirty amplification cycles were performed at $94^{\circ} \mathrm{C}$ for $30 \mathrm{sec}, 55^{\circ} \mathrm{C}$ for $30 \mathrm{sec}$, and a final extension for $5 \mathrm{~min}$ at $72^{\circ} \mathrm{C}$.

Detection of the insertion copy number. It has been reported that multiple copies of the transgene may integrate following electroporation and that the gene dosage may affect the expression levels of the recombinant protein (15). Therefore, in order to achieve a more physiologically comparable result, quantitative PCR (qPCR) was used to determine the copy number of the transgenes in the positive transformants. The $h I F N-\gamma$ insertion copy number was examined three times for each positive clone. Data were normalized against the ACT1 gene as an endogenous control (housekeeping gene). The primers used were: hIFN- $\gamma$, forward: 5'-AGGAAGAGTCCGATAGAAAG-3' and reverse: 5'-TCAGCCATGACCTGGATTAA-3'; ACT1, forward: 5'-TCATGGTCGGTATGGGTCAA-3' and reverse: 5'-ACGATACCGTGCTCGATTGG-3'.

Genomic DNA was used as the template for qPCR analysis. Reaction conditions were established according to the $\mathrm{SYBR}^{\circledR}$ Premix Ex Taq ${ }^{\mathrm{TM}}$ II manual (Takara). Each $20-\mu$ l reaction contained $10 \mu \mathrm{l} \mathrm{SYBR}$ Premix Ex Taq II (2X), $10 \mu \mathrm{M}$ forward and reverse primers (1 $\mu \mathrm{l}$ each), $2 \mu \mathrm{l}$ sample genomic DNA and $6 \mu \mathrm{l}$ sterile double deionized water. qPCR reactions were run in triplicate on ABI7000 (Applied Biosystems, Foster City, CA, USA) under the following conditions: $95^{\circ} \mathrm{C}$ for $30 \mathrm{sec}$, followed by 40 cycles of $95^{\circ} \mathrm{C}$ for $5 \mathrm{sec}$ and $60^{\circ} \mathrm{C}$ for $30 \mathrm{sec}$. The amplicon specificity was verified by analyzing the melting curve after 40 cycles and by agarose gel electrophoresis.

Expression of hIFN- $\gamma$ in P. pastoris. Single copy clones were placed in $10 \mathrm{ml}$ buffered glycerol-complex (BMGY) medium (1.0\% yeast extract, $2.0 \%$ peptone, $1.34 \%$ yeast nitrogen base, $4 \times 10^{-5}$ biotin, $100 \mathrm{mM}$ potassium phosphate, $\mathrm{pH} 6.0$, and $1.0 \%$ glycerol) and incubated at $30^{\circ} \mathrm{C}$ for $24 \mathrm{~h}$. Subsequently, the cells were harvested by centrifugation for $5 \mathrm{~min}$ at $1500 \mathrm{x} \mathrm{g}$ and resuspended in $10 \mathrm{ml}$ buffered methanol-complex (BMMY) medium (BMGY medium with $0.5 \%$ methanol instead of $1.0 \%$ glycerol). Methanol was added to a final concentration of $0.5 \%(\mathrm{v} / \mathrm{v})$ every $24 \mathrm{~h}$. To detect the expression of rhIFN- $\gamma$ in the supernatant, $12 \%(\mathrm{w} / \mathrm{v})$ Tricine-SDS-PAGE, western blot analysis and amino-terminal sequencing were performed. Tricine-SDS-PAGE analysis was performed using a $12 \%$ gel according to the protocol proposed by Schägger (16).

For western blot analysis, proteins were transblotted onto a polyvinylidene fluoride (PVDF) membrane. The membrane was blocked using 5\% BSA in Tris-buffered saline with Tween (TBST) (150 mM NaCl, $25 \mathrm{mM}$ Tris, and 0.05\% Tween-20, $\mathrm{pH} 7.5$ ) for $2 \mathrm{~h}$ at room temperature, and subsequently incubated with mouse anti-HSA monoclonal antibody at $4^{\circ} \mathrm{C}$ overnight. After being washed three times with TBST, the membrane was incubated with goat anti-mouse IgG conjugated to horseradish 
peroxidase (HRP) (Beijing Dingguo Biotechnology Company) for $2 \mathrm{~h}$ at room temperature. Finally, the membrane was washed with TBST and proteins were detected with 3,3'-diaminobenzidine (DAB).

The amino-terminal sequence of rhIFN- $\gamma$ was determined using automated Edman degradation performed on a model PPSQ-21A protein sequencer (Shimadzu, Japan).

Purification of $r h I F N-\gamma$. To purify rhIFN- $\gamma$ from the culture supernatant, 5 liters of $P$. pastoris culture were centrifuged at $1,500 \mathrm{x} \mathrm{g}$ for $15 \mathrm{~min}$. As the molecular weight of rhIFN- $\gamma$ is $17 \mathrm{kDa}$, proteins in the supernatant with a molecular weight of 10-50 kDa were isolated and concentrated using Vivaflow 200 PES 50,000 MWCO and PES 10,000 MWCO. The concentrated sample was loaded onto the SP Sepharose XL for further separation, as the isoelectric point of hIFN- $\gamma$ is $\sim 8.1-9.1$. The rhIFN- $\gamma$ fraction was concentrated by Vivaspin $2 \mathrm{ml}$. Fractions of each step were analyzed on 15\% Tricine-SDS-PAGE. The rhIFN $-\gamma$ fraction was stored at $-20^{\circ} \mathrm{C}$. Protein concentration was determined using a Bradford protein assay kit. The purity of rhIFN- $\gamma$ was determined by HPLC analysis.

Activity assay of $h I F N-\gamma$. The antiviral activity of rhIFN- $\gamma$ was evaluated as previously described (17). The human carcinoma cell line, HEP2C, was challenged with the encephalomyocarditis virus (EMCV) in the presence or absence of hIFN- $\gamma$. Antiviral activity was calculated in comparison with the standard obtained from Novoprotein.

\section{Results}

Construction of expression plasmids and transformation of $P$. pastoris. The 516-bp gene fragment encoding hIFN- $\gamma$ in addition to the BspT1041 and $K p n \mathrm{I}$ sites was synthesized by Sangon Biotechnology Co. and subcloned into pPICZ $\alpha \mathrm{C}$ in order to generate the recombinant plasmid, pNhIFN. The 451-bp gene fragment, encoding the mature hIFN- $\gamma$ in addition to the $X h o \mathrm{I}$ and $\mathrm{XbaI}$ sites, was amplified by PCR from pNhIFN and subcloned into pPICZ $\alpha \mathrm{C}$ to generate the recombinant plasmid, poIFN. Nucleotide sequencing analysis confirmed that the plasmids contained the correct $h I F N-\gamma$ gene (data not shown).

In order to integrate $h I F N-\gamma$ into the genome of P.pastoris by homologous recombination, the plasmids were linearized with $\mathrm{SacI}$ and transformed into electrocompetent $P$. pastoris cells. Successful integration was confirmed by PCR using AOX1 universal primers. The PCR results revealed that in positive yeast transformants there was one band of $\sim 787 \mathrm{bp}$ (pNhIFN) or $948 \mathrm{bp}$ (paIFN). The cells transformed with a pPICZ $\alpha \mathrm{C}$ empty plasmid were used as a negative control, and in these cells one band of $\sim 588$ bp was identified. qPCR data indicated that $90 \%$ of transformants contained a single copy of the gene, with only single-copy integrants being selected for the comparison experiments.

Expression and detection of rhIFN- $\gamma$ in P.pastoris. Single-copy clones of each strain were inoculated in BMGY medium for $24 \mathrm{~h}$ and induced in BMMY medium for the expression of rhIFN- $\gamma$. Each strain was analyzed for rhIFN- $\gamma$ expression on Tricine-SDS-PAGE following $96 \mathrm{~h}$ of induction. Fig. 1A shows
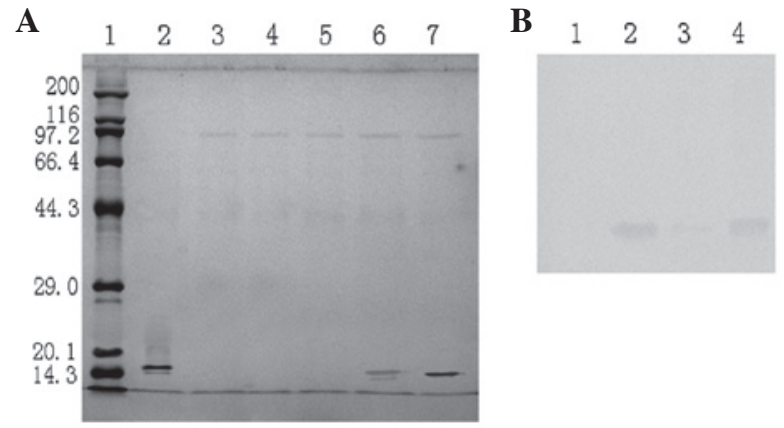

Figure 1. (A) Coomassie-stained Tricine-SDS-PAGE profile of Pichia pastoris (P. pastoris) clones expressing recombinant human interferon- $\gamma(\operatorname{rhIFN}-\gamma)$. Lane 1, broad molecular weight protein marker (6.5-200 kDa); lane 2, standard human interferon- $\gamma($ hIFN- $\gamma$ ); lane 3 , supernatant of the P.pastoris strain transformed with pPICZ $\alpha \mathrm{C}$; lanes 4 and 5 , supernatant of the $P$. pastoris strain transformed with pNhIFN, containing the IFN- $\gamma$ gene with the native secretion signal peptide; lanes 6 and 7, supernatant of the P.pastoris strain transformed with p $\alpha$ IFN, containing the $I F N-\gamma$ gene with the MF- $\alpha$ signal peptide. (B) Western blot analysis of the recombinant $P$. pastoris supernatant expressing hIFN- $\gamma$. Lane 1 , supernatant of the P. pastoris strain transformed with pPICZ $\alpha \mathrm{C}$; lane 2 , standard hIFN- $\gamma$; lane 3 , supernatant of the $P$. pastoris strain transformed with pNhIFN; lane 4, supernatant of the P.pastoris strain transformed with p $\alpha$ IFN. The Coomassie staining profile of the $P$. pastoris strain transformed with pNhIFN did not present a band at $15 \mathrm{kDa}$, but a faint band was detected by western blotting.

a Coomassie-stained Tricine-SDS-PAGE of supernatants collected from induced $P$. pastoris cultures transformed with the expression plasmids of the pPICZ $\alpha \mathrm{C}$ control, pNhIFN or paIFN. The supernatant of the control strain (lane 3) did not reveal any band corresponding to the standard rhIFN- $\gamma$ (lane 2). The supernatant of the P. pastoris strain transformed with pNhIFN (lanes 4 and 5), containing the $h I F N-\gamma$ gene with the native secretion signal peptide, did not reveal a rhIFN- $\gamma$ protein band. The supernatant of the P. pastoris strain transformed with paIFN (lanes 6 and 7), containing the $h I F N-\gamma$ gene with the MF- $\alpha$ signal peptide, showed one protein band that was slightly higher than the hIFN- $\gamma$ band.

Western blot analysis using an anti-human IFN- $\gamma$ antibody confirmed that the protein band from the supernatant of the p $\alpha$ IFN-transformed strain was hIFN- $\gamma$ (Fig. 1B, lane 4). However, it showed a faint band in the supernatant of the pNhIFN-transformed P. pastoris strain (Fig. 1B, lane 3). These results suggest that the native hIFN- $\gamma$ secretion signal peptide is capable of secreting particularly low levels of hIFN- $\gamma$ that may only be detected by western blot analysis, as it is more sensitive than Coomassie staining Tricine-SDS-PAGE.

Therefore, only the MF- $\alpha$ signal peptide cleavage site of the recombinant rhIFN- $\gamma$ was examined, and $\mathrm{N}$-terminal sequencing revealed that the first 14 amino acids of rhIFN- $\gamma$ were QDPYVKEAENLKKY, which were identical to the $\mathrm{N}$-terminal sequence of hIFN- $\gamma$, thus confirming the successful expression of the protein.

Purification and biological activities of rhIFN- $\gamma$ in P.pastoris. Following the isolation and concentration of rhIFN- $\gamma$ from the $P$. pastoris cultures, $\operatorname{rhIFN}-\gamma$ was then purified with cation exchange chromatography (SP Sepharose XL), using an AKTA Explorer 100 chromatography system. The optimal $\mathrm{pH}$ of the $50 \mathrm{mM}$ acetate buffer for binding was 5.0 and the optimal $\mathrm{NaCl}$ (Beijing Dingguo Biotechnology Company) 
Table I. Summary of purification steps of recombinant human interferon- $\gamma$ (rhIFN- $\gamma$ ) from Pichia pastoris.

\begin{tabular}{lccccc}
\hline Purification steps & Volume $(\mathrm{ml})$ & Total protein $(\mathrm{mg})$ & rhIFN- $\gamma(\mathrm{mg})$ & Recovery $(\%)$ & Purity (\%) \\
\hline Supernant & 5000 & 4500 & 1500 & & 33 \\
Vivaflow 200 & 100 & 2415 & 1208 & 80.5 & 50 \\
SP Sepharose XL & 50 & 751 & 676 & 56 & 90 \\
Vivaspin 2 ml & 44 & 601 & 574.6 & 85 & 95.6 \\
\hline
\end{tabular}

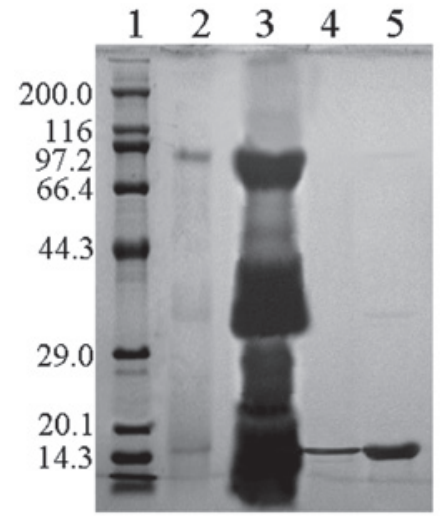

Figure 2. Tricine-SDS-PAGE analysis of purified recombinant human interferon- $\gamma($ (rhIFN $-\gamma)$. Lane 1 , broad molecular weight protein marker (6.5-200 kDa); lane 2, supernatant of the Pichia pastoris strain; lane 3, purified with Vivaflow 200; lane 4, purified with cation exchange chromatography; lane 5, purified with Vivaspin $2 \mathrm{ml}$.

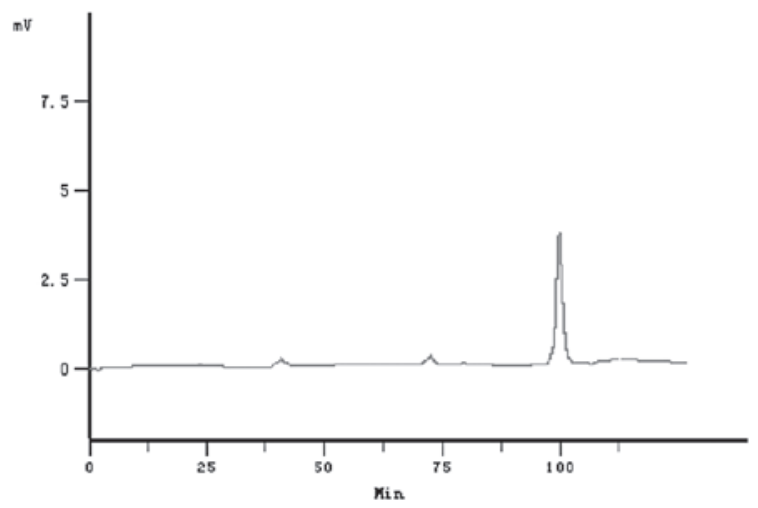

Figure 3. Reversed phase HPLC assay of purified recombinant human interferon- $\gamma($ rhIFN- $\gamma)$. The final highly purified rhIFN- $\gamma$ showed a single peak.

concentration for elution was $0.4 \mathrm{M}$. The rhIFN- $\gamma$ fraction was concentrated by Vivaspin $2 \mathrm{ml}$. Following these procedures, from 5 liters of culture medium, we obtained a total of $800 \mathrm{mg}$ of purified rhIFN- $\gamma$. rhIFN- $\gamma$ achieved a purity of $96.7 \%$ as revealed by Tricine-SDS-PAGE and HPLC (Figs. 2 and 3). Protein recovery and purity of rhIFN- $\gamma$ at the various purification steps are shown in Table I.

To determine the antiviral effects of purified rhIFN- $\gamma$, an antiviral assay was performed by challenging HEP2C cells with EMCV in the presence of varying concentrations of rhIFN- $\gamma$. In three determinations, the calculated specific activity of rhIFN- $\gamma$ ranged from $1 \times 10^{7}$ to $1.6 \times 10^{7} \mathrm{IU} / \mathrm{mg}$ protein, as assessed against the standard (Fig. 4). The results demonstrated

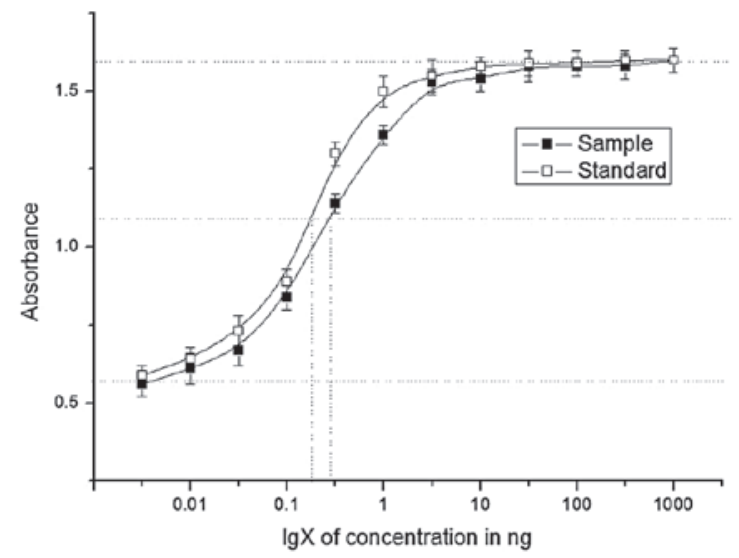

Figure 4. Antiviral assay. Purified recombinant human interferon- $\gamma($ rhIFN- $\gamma)$ activity was assessed against a commercially available standard obtained from Novoprotein. The assay involved challenging HEP2C cells with encephalomyocarditis virus (EMCV) in the presence or absence of rhIFN- $\gamma$. The concentration yielding a 50\% increase in absorbance between the baseline and maximum plateau absorbance values (marking a 50\% reduction in cytopathic effect) defined the assay endpoint. The calculated specific activities of the prepared rhIFN- $\gamma$ ranged from $1 \times 107$ to $1.6 \times 107 \mathrm{IU} / \mathrm{mg}$ protein following three determinations. The graph is of a representative experiment. -, Novoprotein standard (specific activity $1.5 \times 107 \mathrm{IU} / \mathrm{mg}$ protein); $\square$, purified rhIFN- $\gamma$ (specific activity $1.2 \times 107 \mathrm{IU} / \mathrm{mg}$ protein).

that rhIFN- $\gamma$ produced by $P$. pastoris had lower levels of biological activity compared with the standard obtained from Novoprotein, however, the simpler purification strategy makes this a useful method.

\section{Discussion}

hIFN- $\gamma$ is a cytokine with multiple biological and pharmaceutical functions $(2,18)$, thus it is expressed in a variety of systems, particularly in E. coli and $\mathrm{CHO}$ cells. The disadvantage of $E$. coli is that the accumulation of rhIFN- $\gamma$ forms inactive and insoluble aggregates known as inclusion bodies. Although a variety of techniques have been developed to produce active recombinant proteins (dilution, diafiltration, urea, combined urea-ammonium chloride, chaperones, detergent-assisted and chromatography refolding), the complex procedures may lead to a low yield of the active product $(10,19-21)$. Furthermore, rhIFN- $\gamma$ expressed in $E$. coli is different from native mature hIFN- $\gamma$, as its N-terminal amino acid is methionine, which may have clinical and pharmaceutical side effects. The disadvantage of the $\mathrm{CHO}$ cell system is the low productivity of rhIFN- $\gamma$. Although culturing recombinant $\mathrm{CHO}$ cells at $32^{\circ} \mathrm{C}$ may increase the productivity of rhIFN- $\gamma$, it is $<10 \mathrm{mg} / 1$. In our study, we used the $P$. pastoris system to express rhIFN- $\gamma$ 
due to of the following features: low cost, high productivity, a strong regulated AOX1 promoter, growth to a high cell density in a simple medium and stable integration of heterogeneous genes into the genome. Furthermore, $P$. pastoris secretes few endogenous proteins to the culture medium, making purification of recombinant proteins easier.

In the $P$. pastoris expression system, the secretion signal peptide is essential for the successful expression of heterologous proteins. For IFN- $\alpha 2 b$ expression, the native secretion signal of IFN- $\alpha 2 b$ did not secrete the protein, but the $\alpha$-prepro sequence without the EAEA repeats secreted $200 \mathrm{mg} / 1 \mathrm{IFN}-\alpha 2 \mathrm{~b}$ into the culture medium (22). Expression of human serum albumin, using its native signal peptide, has been reported to produce $>1 \mathrm{~g} / \mathrm{l}$ (23). For the secretory efficiency of recombinant xylanase $\mathrm{xyn} B$, the bovine $\beta$-casein signal peptide was less efficient than the $\alpha$-factor prepro sequence (24). Polygalacturonase genes were expressed in $P$. pastoris with the native signal peptide or the $\alpha$-factor secretion signal peptide, but only slight differences in expression were observed (25). As signal peptides secrete recombinant proteins with varying efficiencies, it is important to examine different signal peptides that secrete the same protein in $P$. pastoris. In this study, hIFN- $\gamma$ was successfully expressed in $P$. pastoris using either the native signal peptide of hIFN- $\gamma$ or the $\alpha$-factor signal peptide, although the former mediated trace levels of rhIFN- $\gamma$ secretion, while the latter mediated secretion of $300 \mathrm{mg} / \mathrm{l} \mathrm{rhIFN}-\gamma$ into the culture medium with the same amino terminal sequence as that of the native protein.

The biologically active form of hIFN- $\gamma$ is a homodimer that interacts with a heterodimeric receptor consisting of INF- $\gamma$ receptor 1 and 2 (26,27). In our study, the activity of rhIFN- $\gamma$ was only slightly lower than that of the hIFN- $\gamma$ standard, suggesting that the majority of the rhIFN- $\gamma$ dimerized following the correct secretion into the culture, while small quantities of the rhIFN- $\gamma$ may have remained in the monomer form or formed other complex structures. In conclusion, results of this study demonstrate that large quantities of biologically active rhIFN- $\gamma$ may be produced from $P$. pastoris.

\section{Acknowledgements}

The authors would like to thank Jilin Department of Health, Jilin, China, for their financial support (no. 2011I087).

\section{References}

1. Wheelock EF: Interferon-like virus-inhibitor induced in human leukocytes by phytohemagglutinin. Science 149: 310-311, 1965.

2. Chen J and Liu X: The role of interferon gamma in regulation of $\mathrm{CD}^{+} \mathrm{T}$-cells and its clinical implications. Cell Immunol 254: 85-90, 2009.

3. Billiau A and Matthys P: Interferon-gamma: a historical perspective. Cytokine Growth Factor Rev 20: 97-113, 2009.

4. Suárez-Méndez R, García-García I, Fernández-Olivera N, Valdés-Quintana M, Milanés-Virelles MT, Carbonell D, Machado-Molina D, Valenzuela-Silva CM and López-Saura PA: Adjuvant interferon gamma in patients with drug-resistant pulmonary tuberculosis: a pilot study. BMC Infect Dis 4: 44, 2004.

5. Chang TT and Stevens SR: Atopic dermatitis: the role of recombinant interferon-gamma therapy. Am J Clin Dermatol 3: 175-183, 2002.

6. Parvez MK, Sehgal D, Sarin SK, Basir SF and Jameel S: Inhibition of hepatitis B virus DNA replicative intermediate forms by recombinant interferon-gamma. World J Gastroenterol 12: 3006-3014, 2006.
7. Xu R, Ying B, Zhao S, Li C and Wang Y: Construction and identification of a recombinant adenovirus which expresses human interferon-gamma. Chin J Biotechnol 13: 1-8, 1997.

8. Arakawa T, Hsu YR and Yphantis DA: Acid unfolding and self-association of recombinant Escherichia coli derived human interferon gamma. Biochemistry 26: 5428-5432, 1987.

9. Zhang Z, Tong KT, Belew M, Pettersson T and Janson JC: Production, purification and characterization of recombinant human interferon gamma. J Chromatogr 604: 143-155, 1992.

10. Mohammadian-Mosaabadi J, Naderi-Manesh H, Maghsoudi N, Nassiri-Khalili MA, Masoumian MR and Malek-Sabet N: Improving purification of recombinant human interferon gamma expressed in Escherichia coli; effect of removal of impurity on the process yield. Protein Expr Purif 51: 147-156, 2007.

11. Fieschko JC, Egan KM, Ritch T, Koski RA, Jones M and Bitter GA: Controlled expression and purification of human immune interferon from high-cell-density fermentations of Saccharomyces cerevisiae. Biotechnol Bioeng 29: 1113-1121, 1987.

12. Chen YJ, Chen WS and Wu TY: Development of a bi-cistronic baculovirus expression vector by the Rhopalosiphum padi virus 5' internal ribosome entry site. Biochem Biophys Res Commun 335: 616-623, 2005.

13. Devos R, Opsomer C, Scahill SJ, Van der Heyden J and Fiers W: Purification of recombinant glycosylated human gamma interferon expressed in transformed Chinese hamster ovary cells. J Interferon Res 4: 461-468, 1984.

14. Tan HK, Lee MM, Yap MG and Wang DI: Overexpression of cold-inducible RNA-binding protein increases interferon-gamma production in Chinese-hamster ovary cells. Biotechnol Appl Biochem 49: 247-257, 2008.

15. Li K, Gao H, Gao L, Qi X, Gao Y, Qin L, Wang Y and Wang X: Recombinant gp90 protein expressed in Pichia pastoris induces a protective immune response against reticuloendotheliosis virus in chickens. Vaccine 30: 2273-2281, 2012.

16. Schägger H: Tricine-SDS-PAGE. Nat Protoc 1: 16-22, 2006.

17. Reddy PK, Reddy SG, Narala VR, Majee SS, Konda S, Gunwar S and Reddy RC: Increased yield of high purity recombinant human interferon-gamma utilizing reversed phase column chromatography. Protein Expr Purif 52: 123-130, 2007.

18. Zaidi MR and Merlino G: The two faces of interferon-gamma in cancer. Clin Cancer Res 17: 6118-6124, 2011.

19. Yan X, Hu S, Guan YX and Yao SJ: Coexpression of chaperonin GroEL/GroES markedly enhanced soluble and functional expression of recombinant human interferon-gamma in Escherichia coli. Appl Microbiol Biotechnol 93: 1065-1074, 2012.

20. Petrov S, Nacheva G and Ivanov I: Purification and refolding of recombinant human interferon-gamma in urea-ammonium chloride solution. Protein Expr Purif 73: 70-73, 2010.

21. Jin T, Guan YX, Yao SJ, Lin DQ and Cho MG: On-column refolding of recombinant human interferon-gamma inclusion bodies by expanded bed adsorption chromatography. Biotechnol Bioeng 93: 755-760, 2006.

22. Ghosalkar A, Sahai V and Srivastava A: Secretory expression of interferon-alpha $2 \mathrm{~b}$ in recombinant Pichia pastoris using three different secretion signals. Protein Expr Purif 60: 103-109, 2008.

23. Kobayashi K, Kuwae S, Ohya T, Ohda T, Ohyama M, Ohi H, Tomomitsu K and Ohmura T: High-level expression of recombinant human serum albumin from the methylotrophic yeast Pichia pastoris with minimal protease production and activation. J Biosci Bioeng 89: 55-61, 2000.

24. He Z, Huang Y, Qin Y, Liu Z, Mo D, Cong P and Chen Y: Comparison of alpha-factor preprosequence and a classical mammalian signal peptide for secretion of recombinant xylanase xynB from yeast Pichia pastoris. J Microbiol Biotechnol 22: 479-483, 2012

25. Cho IJ, Yeo IC, Lee NK, Jung SH and Hahm YT: Heterologous expression of polygalacturonase genes isolated from Galactomyces citri-aurantii IJ-1 in Pichia pastoris. J Microbiol 50: 332-340, 2012.

26. Ealick SE, Cook WJ, Vijay-Kumar S, Carson M, Nagabhushan TL, Trotta PP and Bugg CE: Three-dimensional structure of recombinant human interferon-gamma. Science 252: 698-702, 1991

27. Sadir R, Forest E and Lortat-Jacob H: The heparan sulfate binding sequence of interferon-gamma increased the on rate of the interferon-gamma-interferon-gamma receptor complex formation. J Biol Chem 273: 10919-10925, 1998. 